\title{
Differences in motor activation of voluntary and reflex cough in humans
}

\author{
D Lasserson, K Mills, R Arunachalam, M Polkey, J Moxham, L Kalra

See end of article for authors' affiliations .....................

Correspondence to: Dr L Kalra, Department of Stroke Medicine, King's College London School of Medicine, Denmark Hill Campus, Bessemer Road, London SE5 9PJ, UK; lalit.kalra@kcl.ac.uk

Received

21 December 2005

Accepted 12 March 2006

Published Online First

6 April 2006

\begin{abstract}
Objectives: To study motor activation patterns of voluntary and reflex cough adjusted for cough flow rates. Methods: Surface electromyography (EMG) and cough flow rate were measured in 10 healthy volunteers. Voluntary cough was assessed for 20 efforts in each quintile of increasing cough flow rate. Reflex cough was assessed for 25 efforts produced by nebulised L-tartaric acid. EMG was recorded over the expiratory (rectus abdominis, obliques, lower intercostals) and accessory (trapezius, pectoralis major, deltoid, latissimus dorsi) muscles. EMG activity, burst duration and onset were compared for each quintile of voluntary cough, and between voluntary and reflex cough matched for cough flow rate.

Results: EMG activity and burst duration of expiratory and accessory muscles during voluntary cough increased in proportion to cough flow. Expiratory muscles had longer EMG burst duration (difference $68 \mathrm{~ms}(95 \% \mathrm{Cl} 34$ to 102), $\mathrm{p}<0.01$ ) and earlier onset of EMG activity (difference $44 \mathrm{~ms}(95 \% \mathrm{Cl} 20$ to 68 ), $p<0.0001$ ) compared with accessory muscles. EMG activity in all muscles was increased (mean $110.2 \% \vee 56.1 \%, p<0.001$ ) and burst duration (mean $206 \mathrm{~ms} \vee 280 \mathrm{~ms}, \mathrm{p}=0.013$ ) decreased in reflex cough compared with voluntary cough of equal flow rate. There were no differences in EMG onset (difference $8 \mathrm{~ms}(95 \% \mathrm{Cl} 25$ to -9$)$ or burst duration (difference $27 \mathrm{~ms}(95 \% \mathrm{Cl} 58$ to -4 ) between expiratory and accessory muscles.

Conclusions: Functional organisation of motor activity differs between voluntary and reflex cough. Voluntary cough is characterised by sequential activation whereas reflex cough is associated with early and simultaneous activation of expiratory and accessory muscles.
\end{abstract}

$\mathrm{P}$ atients with neuromuscular diseases are uniquely susceptible to respiratory complications, not only because of increased prevalence of aspiration but also because of reduced effectiveness of airway clearing mechanisms. ${ }^{1}$ Of these, cough-whether voluntary or reflex-is particularly important because it generates high expiratory laminar flow in the smaller airways that dislodge and eject foreign material from the pulmonary system. ${ }^{23}$ Many studies have shown that ineffective cough production is associated with a higher prevalence of respiratory complications in neurological diseases. ${ }^{4-8}$ Despite the importance of cough in airway protection, few studies have been undertaken in human subjects to understand the neuromuscular mechanisms involved in the generation of different types of cough.

Much of our current knowledge of motor events during cough production has been inferred from animal experiments. ${ }^{9-16}$ However, voluntary cough cannot be studied in animals, ${ }^{17}$ and reflex cough generated by mechanical or chemical stimulation of airways does not simulate physiological conditions in humans. ${ }^{113-16}$ Many studies were undertaken in anaesthetised animals, which may alter cortical input or depress spinal reflexes and are unrepresentative of neuromuscular events in awake human subjects. ${ }^{17}$ Nevertheless, these studies showed that anterolateral abdominal muscles have a major role in cough generation, ${ }^{15}$ but pectoral muscles may also contribute to cough. ${ }^{12}{ }^{14}$

The importance of abdominal muscles in cough production was confirmed by electromyography (EMG) studies in humans, which showed activation of the rectus abdominis, internal oblique, external oblique, and transversus abdominis muscles during voluntary cough. ${ }^{18} 19$ Abdominal muscle activation was proportional to cough flow rate, and a linear relationship has been demonstrated between increases in EMG activity and voluntary cough flow rates. ${ }^{18-21}$ In addition to abdominal muscles, EMG bursts over the latissimus dorsi and pectoralis major muscles have been demonstrated during voluntary cough efforts in healthy volunteers and tetraplegic subjects in whom corticospinal abdominal inputs were interrupted. $^{22} 23$

Despite studies mapping different muscles involved in cough production, motor programmes underlying voluntary or reflex cough have not been investigated. It is possible that muscle activation patterns for the brainstem mediated reflex cough, which is characterised by rapid generation of high intrathoracic pressures and forceful expulsion of air in response to noxious stimuli, ${ }^{24}$ are different from those associated with voluntary cough in response to stimulation of receptors in the laryngeal mucosa, nervousness or spoken command, which can be initiated and modulated by cortical inputs. ${ }^{25}$ Understanding motor mechanisms which underlie different types of cough may be clinically significant in developing techniques to enhance airway clearing mechanisms in patients with neurological diseases (such as chronic stroke, Parkinson's disease, Alzheimer's disease, and motor neurone disease) who are at increased risk of aspiration. ${ }^{5} 68202627$

The objective of this study was to study motor activation of various muscle groups during voluntary cough in healthy subjects and to compare the patterns of motor activation between voluntary and reflex cough after matching for cough flow rates.

\section{METHODS}

\section{Subjects}

Ten healthy volunteers (seven men) of mean age 34.6 years (range 29-54) were included in the study after giving informed consent. None of the participants had previously participated in similar studies or were known to have pulmonary or neurological disease, chest injury, or had 

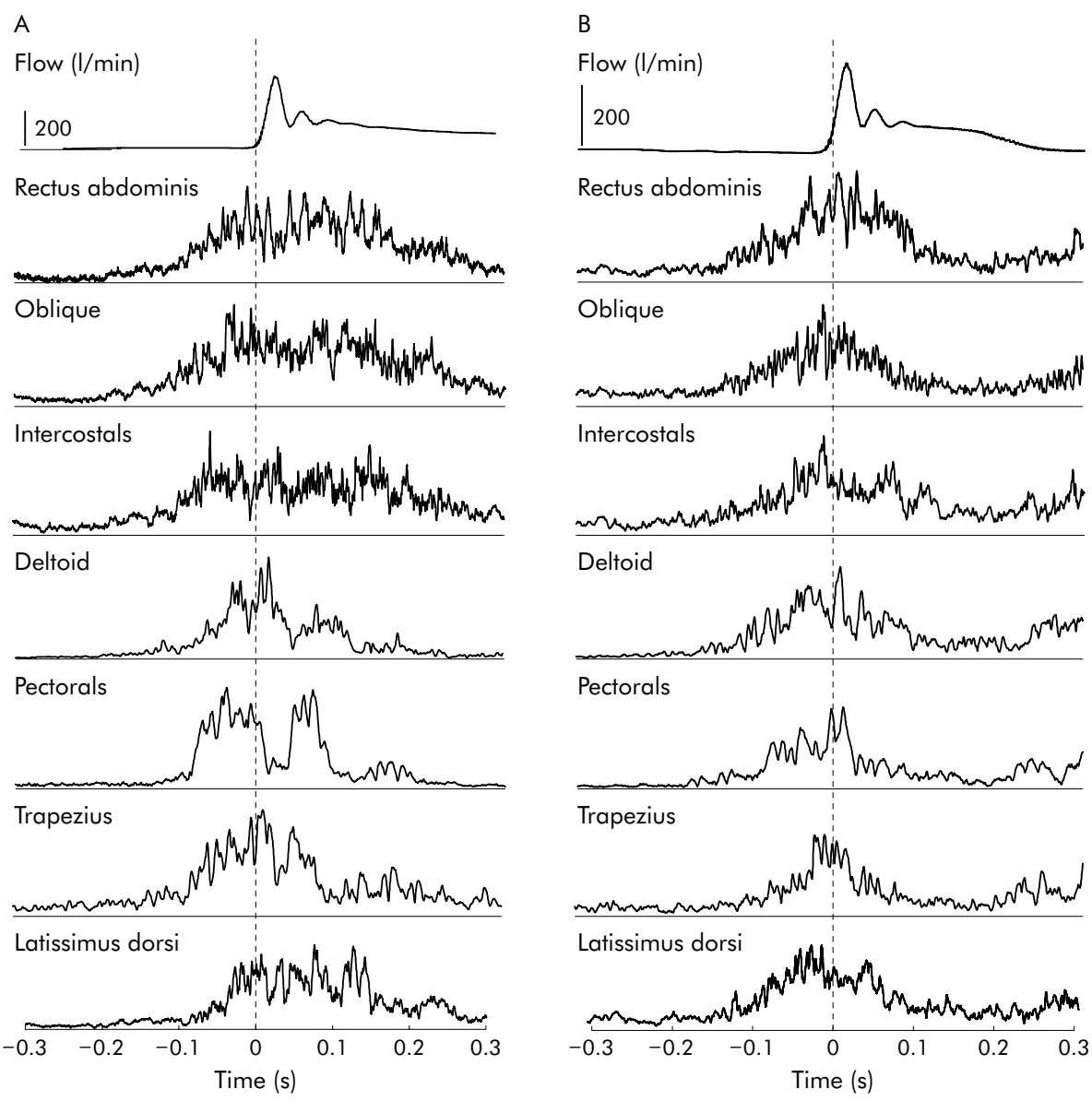

Figure 1 (A) Average flow curve and EMG signals during voluntary cough in a single subject. EMG signals were collected relative to the time of flow onset (time zero) and 10 trials were rectified, averaged, and smoothed. (B) Average flow curve and EMG signals during reflex cough in the same subject as in (A). EMG signals were collected relative to the time of flow onset (time zero) and 10 trials were rectified, averaged, and smoothed. Note the EMG signals show a rise after 0.2 seconds because, in this subject, pairs of coughs were induced.

suffered from respiratory tract infections in the preceding 6 weeks. All subjects had normal respiratory function. Ethical approval was granted by the research ethics committee of King's College Hospital, London.

\section{Procedures}

All procedures were undertaken in a quiet room with subjects seated upright in a comfortable position in a temperature controlled environment.

\section{Measurement of cough flow rates}

Laminar cough flow rates were measured using a standard non-invasive ventilation face mask with "non-leak" mouth seal connected to a Fleisch pneumotachograph by a $30 \mathrm{~cm}$ brass tube. A two-point calibration of the pneumotachograph was undertaken before each test session using a mechanically generated flow of $500 \mathrm{l} / \mathrm{min}$.

Maximum voluntary cough flow rates were measured by asking subjects to perform cough efforts after a deep breath without holding so that cough was initiated from functional residual capacity and measured when flow rates reached a plateau. ${ }^{8}$ The highest values of cough flow rates achieved were used to calculate quintile ranges of increasing flow rate for individual subjects. Visual feedback of the flow for each cough effort was given to subjects using a monitor. Subjects were then asked to perform at least 20 coughs within each of the five quintiles of cough flow rates, guided by visual feedback and separated by periods of rest to prevent fatigue.

Reflex cough was evoked using nebulised sterile solutions of pharmaceutical grade L-tartaric acid delivered via a side port into the connecting tubing of the face mask. Nebulisation was undertaken for 1 minute each using increasing concentrations (5\%, 10\%, 15\%, 20\% and 25\%) until a plateau in the reflex cough flow rate was achieved..$^{26} \mathrm{~A}$ total of 25 reflex coughs were recorded over a time frame of 1.5 seconds each. In 8 of the 10 subjects tartaric acid evoked a second paired cough which was also recorded.

\section{EMG measurement}

The skin surface was prepared using alcohol rub and Neuroline gel (Ambu, MD, USA). Ag-AgCl surface EMG electrodes were positioned on the left side of the body over the trapezius (midpoint between C7 spinal process and acromion, reference on the acromion), pectoralis major $(5 \mathrm{~cm}$ directly below midpoint of the clavicle, reference on the clavicle), deltoid ( $5 \mathrm{~cm}$ below the acromion process, reference on the acromion), latissimus dorsi (mid point on the muscle belly of the posterior aspect of the posterior wall of the axilla, reference on the scapular spine), lower intercostal muscles (8th intercostal space in the mid-axillary line, reference $5 \mathrm{~cm}$ lateral on the 10th rib), external and internal oblique muscles ( $5 \mathrm{~cm}$ directly below the costal margin on a line drawn down to the anterior superior iliac spine, reference placed $5 \mathrm{~cm}$ 

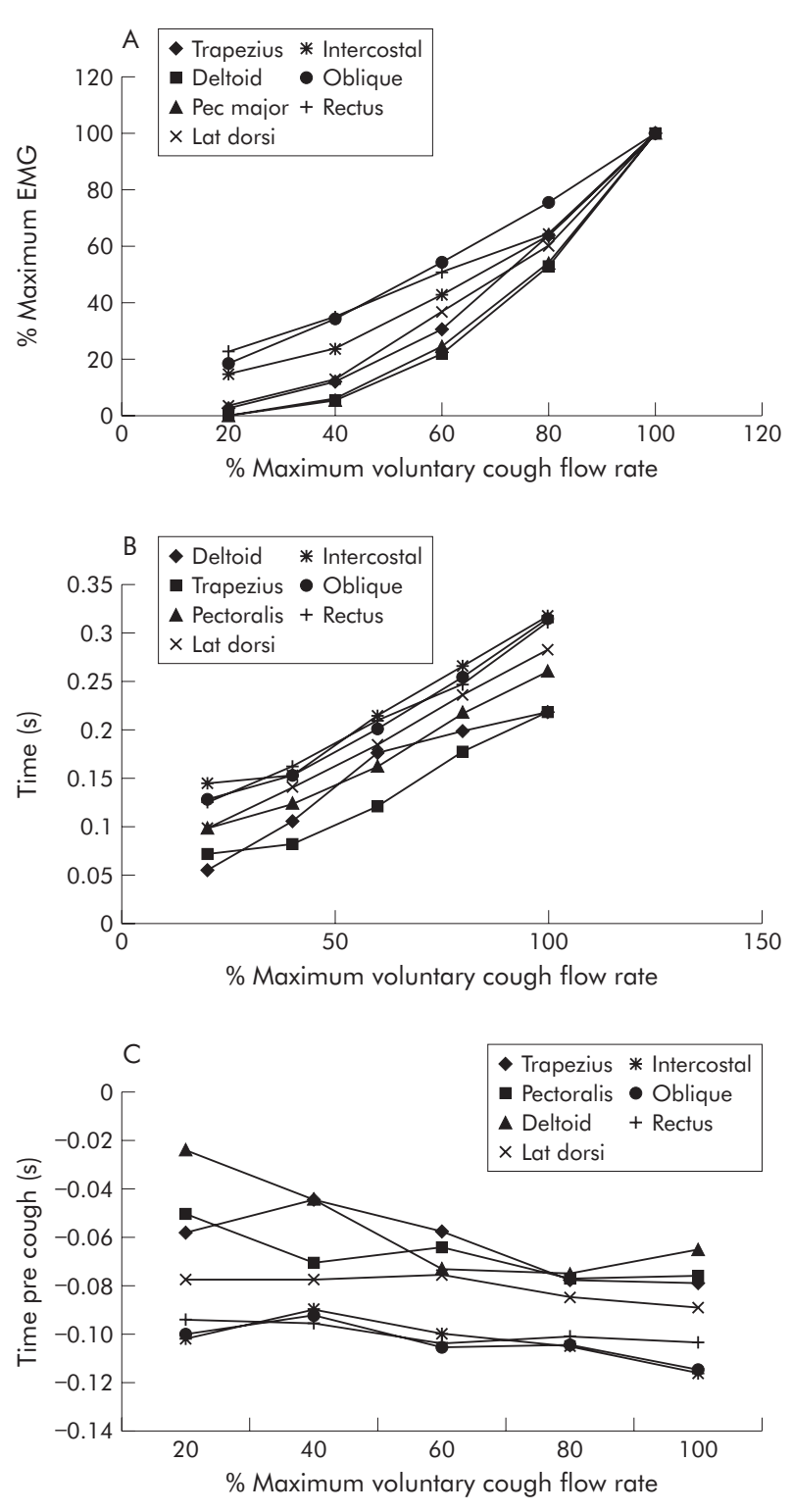

Figure 2 (A) Mean EMG activity, (B) burst duration, and (C) time of onset associated with voluntary cough effort in 10 patients.

medially), and the rectus abdominis $(5 \mathrm{~cm}$ lateral to the umbilicus with the reference a further $5 \mathrm{~cm}$ laterally). In one subject surface recordings and needle EMG recordings were made simultaneously and showed matching traces.

Surface EMG electrodes were connected to a Nicolet Viking IVD EMG recording machine (Nicolet, WI, USA). Signals were amplified and filtered between $10 \mathrm{~Hz}$ and $1 \mathrm{kHz}$ before being passed to an analogue to digital converter (Model CED1401plus, Cambridge Electronics, Cambridge, UK). Signal output from the Fleisch pneumotachograph was amplified by a sensor interface (Validyne, CA, USA) before also being digitalised. A Neurolog circuit (Digitimer, Welwyn Garden City, UK) was used to trigger the recording using the flow signal at the onset of each cough effort and to synchronise the pneumotachograph signal and EMG data with the onset of each cough. Cough flow and EMG data output were displayed on a PC monitor using Signal 2 software (Cambridge Electronics, Cambridge, UK) which recorded each cough in a separate frame with synchronous flow rate and EMG data.

\section{Data analysis}

Data were analysed using SPSS version 12 software (SPSS, Chicago, IL, USA). The mean peak flow rate over 20 maximal voluntary cough efforts was compared with the mean flow rate of the 25 reflex coughs in each subject. Similar comparisons were undertaken for the highest flow rate achieved in any one voluntary effort and any one reflex cough in each subject. Normality of data distribution for airflow, EMG activity, and EMG duration in each quintile of peak cough flow rate was confirmed using the KolmogorovSmirnov test. Data are presented as means with standard deviation and comparisons were undertaken using the $t$ test or the $z$ test as appropriate.

EMG signals from each muscle were rectified, averaged, and then digitally 5-point smoothed. The mean EMG level in the $200 \mathrm{~ms}$ time frame from the start of the sweep was used to define EMG burst onset, area, and duration. Cursors were placed where the EMG activity exceeded the mean $\pm 2 \mathrm{SD}$ baseline level and where it returned to this level. EMG data are presented as mean (SD). EMG comparisons across different flow rates of voluntary cough and between voluntary and reflex cough of equivalent flow rates were undertaken using one way analysis of variance. Statistically important results from this analysis were investigated further by three pairwise tests for each ANOVA and adjusted for multiple testing using the Bonferroni correction. EMG data for voluntary cough were analysed by calculating quintiles of voluntary cough flow rates using Signal software. EMG was rectified and then averaged for each quintile, removing any with cardiac artefacts. At each quintile of voluntary cough flow rate the onset of the averaged EMG burst (relative to time of the onset of cough), EMG burst duration and total EMG activity, defined as the area under the curve (AUC) above baseline voltage between burst onset and offset, were calculated. EMG activity was normalised for each subject by expressing the averaged AUC of EMG at each level of the voluntary cough as a percentage of the averaged AUC of EMG at the top quartile of voluntary cough flow rate for the subject. Muscles were divided into expiratory (rectus abdominis, external and internal oblique, lower intercostals) and accessory (pectoralis major, deltoid, trapezius, latissimus dorsi) groups and their averaged EMG onset and burst duration were compared.

The first and second EMG recordings in each reflex cough pair were averaged separately for the first (R1) and second (R2) reflex cough for each subject. The mean onset time of the EMG burst (relative to time of the onset of cough), mean EMG burst duration, and normalised EMG activity of expiratory and accessory muscles were calculated for Rl and R2 as for voluntary cough. Electrophysiological data for separately averaged R1 and R2 reflex coughs were compared with the quintile of the mean voluntary cough flow rate corresponding to the mean flow rate achieved during reflex cough by each subject. This allowed direct comparisons of onset of the EMG burst (relative to time of the onset of cough), EMG burst duration, and normalised EMG activity at comparable cough flow rates between voluntary cough and reflex cough. The averaged EMG onset and burst duration between the expiratory and accessory group of muscles were compared for reflex cough and also compared between voluntary and reflex cough using paired $t$ tests.

\section{RESULTS}

The mean (SD) peak cough flow rate of voluntary cough was 568 (127) $1 / \mathrm{min}$ and that of the two reflex coughs (Rl and R2) were 370 (156) l/min and 272 (121) l/min, respectively $(\mathrm{p}<0.0001$ for both). The maximal cough flow rate achieved in any one effort was also higher for voluntary cough than for reflex cough (mean difference 146 (95\% CI 70 to 222 ) l/min, 
Table 1 Mean (SD) EMG activity (normalised to the mean value of the top quartile), EMG burst duration (ms), and time between EMG burst onset and cough onset (ms) during voluntary cough at each quintile of cough flow in 10 subjects

\begin{tabular}{|c|c|c|c|c|c|}
\hline & \multicolumn{5}{|c|}{ Flow quintiles } \\
\hline & $0-20 \% *$ & $21-40 \%$ & $41-60 \%$ & $61-80 \%$ & $81-100 \%$ \\
\hline \multicolumn{6}{|l|}{ EMG activity } \\
\hline Rectus abdominis & $22.5(24.9)$ & $34.8(22.5)$ & $50.7(22.5)$ & $64.8(18.2)$ & 100 \\
\hline Int/ext oblique & $18.7(14.9)$ & $33.8(18.4)$ & $54.1(21.8)$ & $75.4(19.5)$ & 100 \\
\hline Intercostal/diaphragm & 15.1 (13.5) & $23.2(16.7)$ & $42.8(17.9)$ & $63.6(20.9)$ & 100 \\
\hline All expiratory muscles & $18.8(17.8)$ & $30.6(19.2)$ & $49.2(20.7)$ & $67.9(19.5)$ & 100 \\
\hline Deltoid & 0.4 & $4.8(12.0)$ & 21.7 (20.9) & $52.3(25.2)$ & 100 \\
\hline Pectoralis major & 0.2 & $6.1(7.0)$ & 24.8 (18.7) & $54.1(30.6)$ & 100 \\
\hline Trapezius & 2.6 & $11.9(21.7)$ & $30.7(29.2)$ & $63.8(33.6)$ & 100 \\
\hline Latissimus dorsi & 3.2 & $13.0(10.4)$ & $36.4(23.1)$ & $60.7(24.1)$ & 100 \\
\hline All accessory muscles & 1.6 & $8.9(12.8)$ & $28.4(22.9)$ & $57.7(28.4)$ & 100 \\
\hline \multicolumn{6}{|l|}{ EMG burst duration } \\
\hline Rectus abdominis & $126(42)$ & $162(51)$ & $209(49)$ & $247(60)$ & $311(69)$ \\
\hline Int/ext oblique & 128 (32) & $152(54)$ & 201 (31) & $255(54)$ & $313(52)$ \\
\hline Intercostal/diaphragm & $144(54)$ & $152(70)$ & $214(41)$ & $266(58)$ & $318(58)$ \\
\hline Expiratory muscles & $133(43)$ & $155(58)$ & $208(40)$ & $256(57)$ & $314(60)$ \\
\hline Deltoid & 55 & $105(46)$ & $176(81)$ & $198(70)$ & $218(84)$ \\
\hline Pectoralis major & 98 & $124(64)$ & 162 (117) & 217 (117) & $260(107)$ \\
\hline Trapezius & 72 & $81(41)$ & $120(64)$ & 177 (69) & $218(82)$ \\
\hline Latissimus dorsi & 99 & $141(38)$ & $184(79)$ & $236(92)$ & $283(75)$ \\
\hline Accessory muscles & 81 & $113(47)$ & $160(85)$ & $228(87)$ & $245(87)$ \\
\hline \multicolumn{6}{|l|}{ EMG burst onset } \\
\hline Rectus abdominis & $-90(35)$ & $-96(35)$ & $-104(30)$ & $-101(28)$ & $-104(25)$ \\
\hline Int/ext oblique & $-100(41)$ & $-93(40)$ & $-106(32)$ & $-100(31)$ & $-115(30)$ \\
\hline Intercostal/diaphragm & $-102(30)$ & $-90(42)$ & $-100(34)$ & $-110(31)$ & $-110(35)$ \\
\hline Expiratory muscles & $-97(35)$ & $-93(39)$ & $-103(32)$ & $-104(30)$ & $-110(32)$ \\
\hline Deltoid & -24 & $-44(29)$ & $-73(30)$ & $-75(34)$ & $-65(51)$ \\
\hline Pectoralis major & -51 & $-71(10)$ & $-65(41)$ & -77 (32) & $-76(34)$ \\
\hline Trapezius & -58 & $-44(26)$ & $-57(33)$ & $-77(45)$ & $-79(34)$ \\
\hline Latissimus dorsi & -77 & $-78(20)$ & $-76(34)$ & $-85(28)$ & $-89(35)$ \\
\hline Accessory muscles & -53 & $-59(21)$ & $-68(35)$ & -78 (35) & -77 (39) \\
\hline
\end{tabular}

Table 2 Comparison of mean (SD) EMG activity, burst duration (ms), and burst onset pre-cough (ms) between reflex cough and the comparable quintile of voluntary cough flow rate

\begin{tabular}{|c|c|c|c|c|}
\hline & Voluntary cough & Reflex cough $(R 1)$ & Reflex cough (R2) & $p$ value \\
\hline $\begin{array}{l}\text { Normalised EMG activity } \\
\text { Rectus abdominis } \\
\text { Int/ext oblique } \\
\text { Intercostal/diaphragm } \\
\text { Deltoid } \\
\text { Pectoralis major } \\
\text { Trapezius } \\
\text { Latissimus dorsi }\end{array}$ & $\begin{array}{l}68.2(16.8) \\
54.1(21.1) \\
62.3(18.1) \\
24.3(22.5) \\
55.6(28.9) \\
60.7(34.2) \\
67.2(33.1)\end{array}$ & $\begin{array}{l}73.8(37.4) \\
79.7(41.6) \\
184.0(411.3) \\
78.6(120.6) \\
136.6(309.7) \\
96.4(92) \\
122.9(236.2)\end{array}$ & $\begin{array}{l}60.6(30.8) \\
51.7(28.4) \\
169.4(342.0) \\
83.7(100.6) \\
183.5(392.2) \\
81.2(73.4) \\
132.7(245.8)\end{array}$ & \\
\hline $\begin{array}{l}\text { EMG burst duration (ms) } \\
\text { Rectus abdominis } \\
\text { Int/ext oblique } \\
\text { Intercostal/diaphragm } \\
\text { Deltoid } \\
\text { Pectoralis major } \\
\text { Trapezius } \\
\text { Latissimus dorsi } \\
\text { Mean expiratory muscles } \\
\text { Mean accessory muscles } \\
\text { Mean difference (exp-acc)* }\end{array}$ & $\begin{array}{l}311(69) \\
313(52) \\
318(58) \\
218(84) \\
260(107) \\
218(82) \\
283(75) \\
314(60) \\
245(87) \\
68(47)\end{array}$ & $\begin{array}{l}213(63) \\
231(66) \\
215(52) \\
182(59) \\
213(81) \\
166(83) \\
207(56) \\
220(60) \\
192(70) \\
27(44)\end{array}$ & $\begin{array}{l}171(63) \\
173(50) \\
197(57) \\
177(75) \\
187(54) \\
151(79) \\
184(67) \\
180(57) \\
175(69) \\
6(31)\end{array}$ & $\begin{array}{l}0.001 \\
0.02 \\
0.005\end{array}$ \\
\hline $\begin{array}{l}\text { EMG burst onset (ms) } \\
\text { Rectus abdominis } \\
\text { Int/ext oblique } \\
\text { Intercostal/diaphragm } \\
\text { Deltoid } \\
\text { Pectoralis major } \\
\text { Trapezius } \\
\text { Latissimus dorsi } \\
\text { Mean expiratory muscles } \\
\text { Mean accessory muscles } \\
\text { Mean difference (exp-acc)* }\end{array}$ & $\begin{array}{l}-104(25) \\
-115(30) \\
-110(35) \\
-65(51) \\
-76(34) \\
-79(34) \\
-89(35) \\
-110(32) \\
-77(39) \\
44(34)\end{array}$ & $\begin{array}{l}-92(26) \\
-96(29) \\
-101(28) \\
-87(44) \\
-109(42) \\
-73(33) \\
-84(40) \\
-96(28) \\
-88(40) \\
8(24)\end{array}$ & $\begin{array}{l}-90(29) \\
-94(29) \\
-95(28) \\
-97(44) \\
-101(34) \\
-84(41) \\
-81(33) \\
-93(29) \\
-90(38) \\
2(27)\end{array}$ & $\begin{array}{l}0.107 \\
0.762 \\
0.0001\end{array}$ \\
\hline
\end{tabular}



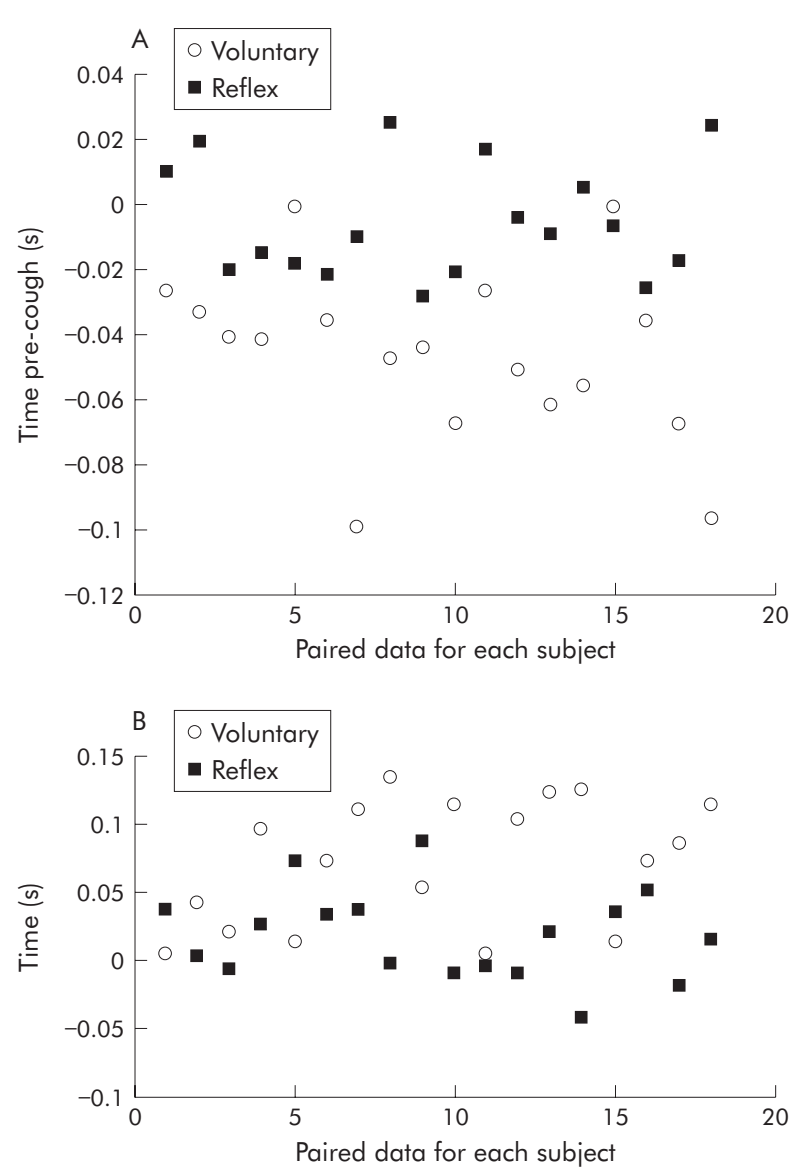

Figure 3 Paired comparisons of (A) the delay in onset of EMG activity and (B) EMG burst duration between expiratory and accessory muscles during reflex cough and voluntary cough matched for cough flow rate.

$\mathrm{p}<0.01)$. The mean duration of the voluntary cough effort was longer than for the reflex cough for both Rl (mean difference $99 \mathrm{~ms}(95 \%$ CI 68 to 130), p<0.005) and R2 (mean difference $114 \mathrm{~ms}(95 \%$ CI 12 to 216$), \mathrm{p}<0.05)$. Although the mean peak cough flow rate of $\mathrm{R} 1$ was greater than $\mathrm{R} 2$ (difference $116 \mathrm{l} / \mathrm{min}$ (95\% CI 68 to 164 ), $\mathrm{p}<0.001$ ), there were no significant differences in cough duration between Rl and R2. Differences between voluntary, R1 and R2 cough flow rates and duration were consistent within individual subjects.

\section{Motor activation in voluntary cough}

All subjects showed similar patterns of EMG and cough flow rate recordings during voluntary cough, a typical example of which is shown in fig lA. Voluntary cough was associated with equal EMG activity in expiratory muscles which increased linearly in proportion with cough flow rate $\left(r^{2}=0.62, \mathrm{p}<0.001\right.$, table 1$)$. In contrast, accessory muscles showed little activity at low cough flow rates but this increased exponentially at high cough flow rates (fig 2A). Mean EMG burst duration increased with cough flow rate for both expiratory and accessory muscles $(\mathrm{p}<0.001$ for both, table 1). Expiratory muscles had longer EMG burst duration than accessory muscles (mean difference 68 ms (95\% CI 102 to 34 ), $\mathrm{p}<0.01$ ) which did not change with increasing muscle activation or cough flow rate (fig $2 \mathrm{~B}$ ). Although onset of EMG activity in all muscle groups preceded cough onset, it was consistently earlier in the expiratory muscles than in the accessory muscles for all flow rates of voluntary cough (mean difference $44 \mathrm{~ms}$ (95\% CI 20 to 68 ), p $<0.0001$, table 1). This difference in the timing of EMG burst onset between expiratory and accessory muscles remained constant across flow rates (fig 2C).

\section{Motor activation in reflex cough}

Motor activation patterns during reflex cough are shown in fig $1 \mathrm{~B}$ and were compared with motor activation patterns of voluntary cough producing the same quintile of cough flow rates in each subject (table 2 ). There were no significant differences in the EMG activation patterns between Rl and R2. Reflex cough was associated with significantly greater mean EMG activity (mean $110.2 \%$ v 56.1\%, p <0.001) but shorter mean EMG burst duration $(206 v 280 \mathrm{~ms}, \mathrm{p}=0.013)$ in all muscle groups compared with voluntary cough of comparable flow rate. In contrast to voluntary cough, EMG activity was significantly greater in accessory than in expiratory muscles (table 2). EMG burst duration of accessory muscles was comparable to the EMG burst duration of expiratory muscles during reflex cough (difference $27 \mathrm{~ms}(95 \% \mathrm{CI}-4$ to 58 ), fig $3 \mathrm{~A}$ ). There were also no differences in the mean duration of EMG burst onset before cough onset between expiratory and accessory muscles during reflex cough (difference $8 \mathrm{~ms}$ (95\% CI -9 to 25), fig $3 \mathrm{~B}$ ). These observations varied significantly from the consistent differences in EMG burst onset and duration between expiratory and accessory muscles seen during voluntary cough $(\mathrm{p}<0.0001$ and $\mathrm{p}<0.005$, respectively $)$.

\section{DISCUSSION}

This study is the first to our knowledge to demonstrate motor sequences involved in the production of voluntary and reflex cough in healthy human subjects. Voluntary cough production was associated with coordinated activation of expiratory and accessory muscles, which showed a graded increase in mean EMG activity and burst duration proportional to cough flow rates produced. Low cough flow rates were produced largely by the activation of expiratory muscles, but accessory muscles became involved sequentially and increasingly for the production of higher flow rates. Reflex cough, on the other hand, was associated with simultaneous onset of EMG activity in expiratory and accessory muscles, increased EMG activity of all muscle groups and shorter EMG burst duration, consistent with rapid and widespread activation of expiratory and accessory muscles in unison. These patterns reflect differences in the functional organisation of muscle activation between voluntary and reflex cough. Sensory inputs from airway afferents in the brainstem mediated reflex cough result in simultaneous efferent outputs to all expiatory and accessory muscles in order to generate maximum airflow for the lung volume at the beginning of the cough. In contrast, cortical inputs to medullary centres (or even bypassing these centres) in voluntary cough can modulate the level and sequence of activation of different muscles and produce more controlled airflows depending upon perceived need. ${ }^{28}$

An important finding of this study was that voluntary cough was associated consistently with EMG activation of shoulder girdle and thoracic (accessory) muscles which increased with increasing cough flow rate. Previous reports support abdominal muscles working as a coordinated unit to generate the high intra-abdominal pressure required for cough, ${ }^{15} 1819$ but there are no reports that extend this functional unit to include accessory muscles. EMG activity of pectoral and intercostal muscles during voluntary cough has been observed inconsistently in previous studies and interpreted as evidence for variability in motor programmes for voluntary cough. ${ }^{22}{ }^{29}$ Evidence from this study supports a common neural drive to functionally related expiratory and accessory muscles which is involved in both reflex and voluntary cough. It also appears that activation of expiratory 
and accessory muscles during voluntary cough can be modulated and integrated, at least in part, by cortical inputs in response to volitional commands or other stimuli and results in graded recruitment of expiratory and accessory muscles to produce cough with high flow rates. This observation has clinical implications for the training of respiratory muscles in patients with chronic neurological or respiratory diseases who may have reduced airway clearance.

One strength of this study is that EMG activity of muscles involved in cough and cough flow rates were measured simultaneously and voluntary and reflex cough were studied in the same subjects. Motor activation was studied for matched flow rates of voluntary and reflex cough and the lowest concentration of tartaric acid required to produce a consistent reflex cough in individual subjects was used to avoid confounding effects of other protective responses at higher concentrations. ${ }^{30}$ Potential sources of bias due to subject variability or methodology were minimised by careful selection of healthy subjects within a relatively narrow age range, predefined protocols for respiratory and EMG measurements, and calibration of equipment before each experimental session.

A limitation of the study was that surface electrodes were used to measure EMG activity. The accuracy of surface EMG may vary because of differences in subcutaneous fat interfering with a good surface EMG signal. However, all subjects were of normal weight for their height and this is an unlikely source of bias. There is a possibility that volume conducted activity in the surface recording of muscles may have prevented selective recordings. More selective recordings could have been undertaken using needle or fine wire electrodes, but the activity, although selective, would not have been representative of the total activity emanating from the muscle, the measurement of which was one of the primary aims of the study. It can be argued that, although EMG recordings may not have been selective for a given muscle, the predominant contribution would be from the muscle closest to the electrodes. As the study compares voluntary with reflex coughs and if the same muscles are assumed to be active in both, then differences in the EMG burst area, duration, and onset can be reasonably attributed to differences in activation pattern rather than volume conducted contamination from other muscles.

The second reflex cough was an unexpected but consistent finding of this study and this pairing of reflex cough efforts has not been described previously. However, there were no significant differences in the EMG activation patterns of Rl and R2. It was not possible to measure lung volumes immediately before reflex cough; differences in lung volumes at cough onset may be partially responsible for lower flow rates seen with reflex cough, especially R2. Low lung volumes also affect muscle length and may affect EMG activation. As the volume of air inspired before acid induced coughs is likely to have been much smaller than for voluntary cough, this may have affected the level of activation of expiratory muscles and the recruitment of accessory muscles. There is no pragmatic method for controlling the volume of air inspired before reflex cough induced by acid inhalation. However, bias due to this is likely to be small because of the similarity of EMG activation patterns for R1 and R2 despite the possibility that lung volumes for R2 would have been smaller than for Rl.

It is probable that reflex cough produced by chemical stimulation under experimental conditions does not simulate clinical conditions in which aspiration occurs. ${ }^{31}$ Inhaled tartaric acid may also stimulate other reflex inputs that interact with cough, and it is possible that this complexity of afferent inputs may have modulated EMG activation. There is an additional afferent component due to accumulated secretions in many lung diseases and muscle activation patterns in supine patients may be very different from healthy subjects sitting upright. However, life threatening situations associated with aspirates of fluid or food which simulate clinical settings cannot be replicated in experimental situations because of safety and ethical considerations. In addition, the study does not investigate neural mechanisms that may underlie differences in muscle activation and there is a possibility that these too may differ between health and disease states.

In conclusion, this study has demonstrated different motor mechanisms underlying voluntary and reflex cough. Although the cough reflex remains the major defence against the risk of aspiration, ${ }^{32}$ sequential activation of expiratory and accessory muscles during voluntary cough is capable of generating high airflows and may offer additional protection in patients with neurological disease who have impaired pharyngeal and laryngeal function. ${ }^{63} 34$ This implies that assessment of voluntary cough should become an integral part of the overall assessment of neurological patients. It may also be possible to develop simple interventions such as voluntary cough exercises in these patients which will help to reduce morbidity and mortality associated with chronic neurological disease. Further research is needed to determine how cough mechanisms are affected in disease and to assess the effectiveness of interventions to improve voluntary cough in clinical settings.

\section{Authors' affiliations}

D Lasserson, L Kalra, Department of Stroke Medicine, King's College London School of Medicine, Denmark Hill Campus, London SE5 9PJ, UK K Mills, R Arunachalam, Department of Clinical Neurophysiology, King's College London School of Medicine, Denmark Hill Campus, London SE5 9PJ, UK

M Polkey, Respiratory Muscle Laboratory, Royal Brompton Hospital, London SW3 6NP, UK

J Moxham, Department of Respiratory Medicine, King's College London School of Medicine, Denmark Hill Campus, London SE5 9PJ, UK

The study was supported by a grant from The Stroke Association, UK (TSA2004/05).

The authors have no financial, academic or personal conflicts of interest to report.

\section{REFERENCES}

1 Polkey MI, Lyall RA, Moxham J, et al. Respiratory aspects of neurological disease. J Neurol Neurosurg Psychiatry 1999;66:5-15.

2 Leith DE, Butler JP, Sneddon SL, et al. Cough. In: Handbook of physiology: the respiratory system. Volume III, Part 1. Bethesda: American Physiological Society, 1990:315-36.

3 Korpas J, Widdicombe JG. Aspects of the cough reflex. Respir Med 1991;85(Suppl A):3-5.

4 Kobayashi $H$, Hosino $M$, Okayama $K$, et al. Swallowing and cough reflexes after onset of stroke. Chest 1994;105:1623.

5 Addington WR, Stephens RE, Gilliland KA. Assessing the laryngeal cough reflex and the risk of developing pneumonia after stroke: an interhospital comparison. Stroke 1999;30:1203-7.

6 Smith-Hammond CA, Goldstein LB, Zajac DJ, et al. Assessment of aspiration risk in stroke patients with quantification of voluntary cough. Neurology 2001;56:502-6.

7 Epstein SK. An overview of respiratory muscle function. Clin Chest Med 1994; 15:619-39.

8 Polkey MI, Lyall RA, Green M, et al. Expiratory muscle function in amyotrophic lateral sclerosis. Am J Respir Crit Care Med 1998;158:734-41.

9 Gilmartin J, Ninane V, De Troyer A. Abdominal muscle use during breathing in the anaesthetised dog. Respir Physiol 1987;70:159-71.

10 De Troyer A, Gilmartin J, Ninane V. Abdominal muscle use during breathing in unanaesthetised dogs. J Appl Physiol 1989;66:20-7.

11 Suzuki H, Kondo T, Yamabayashi H, et al. Influence of central respiratory activity on the cough response in anaesthetized dogs. Jpn J Physiol 1991;41:879-91.

12 Muza S, Criner G, Kelsen S. Effect of lung volume on the respiratory action of the canine pectoral muscles. J Appl Physiol 1992;73:2408-12.

13 Kobayashi I, Kondo T, Suzuki H, et al. Expiratory activity of the inspiratory muscles during cough. Jpn J Physiol 1992;42:905-16. 
14 Bolser DC, Reier PJ. Inspiratory and expiratory patterns of the pectoralis major muscle during pulmonary defensive reflexes. $J$ Appl Physiol 1998;85: 1786-92.

15 Bolser DC, Reier PJ, Davenport PW. Responses of the anterolateral abdominal muscles during cough and expiratory threshold loading in the cat. J Appl Physiol 2000;88:1207-14.

16 Poliaček I, Stránsky A, Jaku J, et al. Activity of the laryngeal abductor and adductor muscles during cough, expiration and aspiration reflexes in cats. Physiol Res 2003:52:749-62.

17 Iscoe S. Control of abdominal muscles. Prog Neurobiol 1998;56:433-506.

18 Strohl K, Mead J, Banzett R, et al. Regional differences in abdominal muscle activity during various manoeuvres in humans. J Appl Physiol: Respirat Environ Exerc Physiol 1981;51:1471-6.

19 Goldman JM, Lehr RP, Millar AB, et al. An electromyographic study of the abdominal muscles during postural and respiratory maneuvers. J Neurol Neurosurg Psychiatry 1987;50:866-96.

20 Fontana GA, Pantaleo T, Lavorini F, et al. Defective motor control of coughing in Parkinson's disease. Am J Respir Crit Care Med 1998;158:458-64.

21 Cox ID, Wallis PJ, Apps MC, et al. An electromyographic method of objectively assessing cough intensity and use of the method to assess effects of codeine on the dose-response curve to citric acid. Br J Clin Pharmacol 1984; 18:377-82.

22 Estenne M, De Troyer A. Cough in tetraplegic subjects: an active process. Ann Intern Med 1990; 1 12:22-8.

23 Fujiwara T, Hara Y, Chino N. Expiratory function in complete tetraplegics: Study of spirometry, maximal expiratory pressure, and muscle activity of pectoralis major and latissimus dorsi muscles. Am J Phys Med Rehabil 1999;78:464-9.
24 Irwin RS, Boulet LP, Cloutier MM, et al. Managing cough as a defense mechanism and a symptom. A consensus panel report of the American College of Chest Physicians. Chest 1998;114(Suppl 2):133-81S.

25 Addington WR, Stephens RE, Widdicombe JG, et al. Electrophysiologic latency to the external obliques of the laryngeal cough expiration reflex in humans. Am J Phys Med Rehabil 2003;82:370-3.

26 Stephens RE, Addington WR, Widdicobe JG. Effect of unilateral middle cerebral artery infarcts on voluntary cough and the laryngeal cough reflex. Am J Phys Med Rehabil 2003;82:379-83.

27 Niimi A, Matsumoto $\mathrm{H}$, Ueda T, et al. Impaired cough reflex in patients with recurrent pneumonia. Thorax 2003;58:152-3.

28 Bolser DC, Davenport PW. Functional organisation of the central cough generation mechanism. Pulm Pharmacol Ther 2202;15:221-5.

29 Chan $\mathrm{CLH}$, Ponsford S, Swash M. The anal reflex elicited by cough and sniff: validation of a neglected clinical sign. I Neurol Neurosurg Psychiatry 2004;75:1449-51.

30 Prudon B, Birring SS, Vara DD, et al. Cough and glottic-stop reflex sensitivity in health and disease. Chest 2005;127:550-7.

31 Fontana GA, Pantaleo T, Lavorini F, et al. A noninvasive electromyographic study on threshold and intensity of cough in humans. Eur Respir J 1997; 10:983-9.

32 Marik PE, Kaplan D. Aspiration pneumonia and dysphagia in the elderly. Chest 2003;124:328-36.

33 Hilker R, Poetter C, Findeisen N, et al. Nosocomial pneumonia after acute stroke: implications for neurological intensive care medicine. Stroke 2003;34:975-81.

34 Taylor $\mathbf{P}$, Tromans A, Harris $\mathrm{K}$, et al. Electrical stimulation of abdominal muscles for control of blood pressure and augmentation of cough in a C3/4 level tetraplegic. Spinal Cord 2002;40:34-6.

\section{LUNG ALERT}

\section{Potential role of Cryptococcus neoformans in the pathogenesis of asthma}

$\Delta$ Goldman DL, Davis J, Bommarito F, et al. Enhanced allergic inflammation and airway responsiveness in rats with chronic Cryptococcus neoformans infection: potential role for fungal pulmonary infection in the pathogenesis of asthma. $J$ Infect Dis 2006;193:1178-86

T

he potential of pulmonary Cryptococcus neoformans infection in immunocompetent subjects to modify allergic inflammation and airway responsiveness was investigated using a rat model.

Rats were inoculated with $C$ neoformans either endotracheally or intravenously. Four modes of infection were studied: short term and persistent localised pulmonary infection, resolved pulmonary infection, and disseminated systemic infection. All were subsequently sensitised and challenged with ovalbumin.

Compared with controls and experimental subjects before sensitisation, only the disseminated infection mode had a higher IgE titre. All active infections had higher bronchoalveolar lavage (BAL) eosinophil counts. After sensitisation and challenge, IgE titre and BAL cell count generally increased. However, when compared with controls, only active localised pulmonary infections showed higher serum titres of total and ovalbumin specific IgE, as well as higher BAL eosinophil counts. Baseline airway resistance did not differ between infected and uninfected rats. However, regardless of sensitisation status, short term pulmonary infected rats had higher airway responsiveness. All forms of active infection expressed increased interleukin (IL)-13, IL-10, and tumour necrosis factor $\alpha$ without any detectable IL-4 or IL-12. Localised infection was associated with higher IL-13 expression than disseminated infection. Furthermore, disseminated (but not localised) infection was associated with an increased level of interferon- $\gamma$.

The authors concluded that active pulmonary cryptococcal infection may enhance allergic response with Th2 polarisation and increased airway responsiveness in rats. They suggested that epidemiological studies are warranted to explore the potential contribution of subclinical cryptococcal infection to the high prevalence of urban asthma.

S A Nachman Assistant Professor on Clinical Medicine, Columbia University/Harlem Hospital Center, New York, NY, USA; san14@columbia.edu 